\title{
Is the Inf-convolution of Law-invariant Preferences Law-invariant?
}

\author{
Peng Liu* Ruodu Wang ${ }^{\dagger} \quad$ Linxiao $\mathrm{Wei}^{\ddagger}$
}

29th January 2020

\begin{abstract}
We analyze the question of whether the inf-convolution of law-invariant risk functionals (preferences) is still law-invariant. In other words, we try to understand whether the representative economic agent (after risk redistribution) only cares about the distribution of the total risk, assuming all individual agents do so. Although the answer to the above question seems to be affirmative for many examples of commonly used risk functionals in the literature, the situation becomes delicate without assuming specific forms and properties of the individual functionals. We illustrate with examples the surprising fact that the answer to the main question is generally negative, even in an atomless probability space. Furthermore, we establish a few very weak conditions under which the answer becomes positive. These conditions do not require any specific forms or convexity of the risk functionals, and they are the richness of the underlying probability space, and monotonicity or continuity of one of the risk functionals. We provide several examples and counter-examples to discuss the subtlety of the question on law-invariance.
\end{abstract}

Key-words: law-invariance, inf-convolution, preferences, risk functionals, risk sharing

\section{Introduction}

The concept of inf-convolution is closely related to the problem of risk sharing and general equilibrium (see e.g. Starr (2011), Delbaen (2012), Rüschendorf (2013) and Embrechts et al. (2018)). In particular, solving for the inf-convolution (or max-convolution) leads to Pareto-optimal allocations in a risk sharing problem with monetary risk measures or utility maximization (see Section 2 for a precise description). In such cases, the inf-convolution serves as the preference functional

${ }^{*}$ Department of Statistics and Actuarial Science, University of Waterloo, Canada. $\square$ peng.liu1@uwaterloo.ca.

${ }^{\dagger}$ Department of Statistics and Actuarial Science, University of Waterloo, Canada. $\square$ wang@uwaterloo.ca.

${ }^{\ddagger}$ College of Science, Wuhan University of Technology, Wuhan, China. $\square$ lxwei@whu.edu.cn. 
of a representative agent in a risk sharing problem with multiple agents. We shall call these preference functionals risk functionals in this paper since we work under the setting that the agents prefer a smaller value of its preference functional over a larger one. In the actuarial literature, inf-convolution serves as a useful tool for solving optimal risk transfer problems in insurance (see e.g. Tsanakas (2009) and Weber (2018)).

We focus on the inf-convolution of law-invariant preference functionals. The property of lawinvariance allows one to reduce the domain of a risk functional from a space of random variables to a corresponding set of distribution functions, providing huge convenience for analysis. Examples of law-invariant functionals include (up to a sign change) mean-variance functionals, expected utilities, rank-dependent utility functionals (Quiggin (1982)), subjective utilities in cumulative prospect theory (Tversky and Kahneman (1992)), and various risk measures and deviation measures as discussed in Artzner et al. (1999), Rockafellar et al. (2006) and Föllmer and Schied (2016). Moreover, many important results or concepts are obtained or defined only for law-invariant functionals, such as the classic notions of risk aversion (Arrow (1974)), the axiomatic characterization of insurance prices (Wang et al. (1997)), the Kusuoka representation (Kusuoka (2001)), the Fatou continuity (Jouini et al. (2006)), ${ }^{1}$ robustness (e.g. Cont et al. (2010)), model uncertainty (e.g. Embrechts et al. (2015)), elicitability (e.g. Ziegel (2016)), and convex level sets (e.g. Weber (2006)).

Given the importance of both law-invariance and inf-convolution, it is natural to ask whether law-invariance is preserved under inf-convolution, the key question we address in this paper. In other words, if all agents in a risk sharing system only care about the distributions of risks, whether the resulting (after risk redistribution) representative agent also only cares about the distribution of the total risk, regardless of how the total risk is defined as a random variable.

In the risk measures literature, the inf-convolution of law-invariant risk measures can sometimes be described analytically, either in an explicitly form or in a dual representation (if convex). In such cases, the explicit form is almost always law-invariant. This includes convex and coherent risk measures (e.g. Jouini et al. (2008), Filipović and Svindland (2008) and Rüschendorf (2013)), quasi-convex risk measures (e.g. Chen et al. (2018)), quantile-type functionals such as the Valueat-Risk (e.g. Embrechts et al. (2018) and Weber (2018)), distortion risk measures (Wang and Wei (2018)), and convex-order consistent risk measures (e.g. Mao and Wang (2016)). These examples are collected in Section 3.3. It is however unclear whether the specific forms and properties of

\footnotetext{
${ }^{1}$ Although the Fatou continuity is defined for general functionals, it is implied by the law-invariance of convex risk measures on $L^{\infty}$, shown by Jouini et al. (2006). Similar results hold on general Orlicz spaces as shown by Gao et al. (2018).
} 
the risk functionals in the above results are essential for such a conclusion to hold with generality. In other words, we aim to study the question of law-invariance with minimal assumptions on the individual risk functionals; in particular, we avoid assuming convexity or additivity in any sense.

The main contributions of the paper are summarized as follows. First, we show the rather surprising fact that, generally, the inf-convolution of law-invariant risk functionals may not be lawinvariant. In an atomless probability space, the construction of a counter-example becomes quite delicate, which we provide in Section 3. In our main results, Theorems 1 and 2 in Section 5, we provide a few weak conditions under which law-invariance of inf-convolution holds in the setting of bounded random variables. Roughly speaking, these conditions require either the underlying probability space to be rich enough in some sense, or the individual risk functionals to satisfy some continuity or monotonicity. Similar results are obtained for $L^{p}$ spaces in Section 7.

As a general message of our main results, in practically relevant cases, one can safely assume law-invariance for the inf-convolution of law-invariant risk functionals. For a concrete example, if one of the risk functionals is a law-invariant monetary risk measure, and the rest are law-invariant, then their inf-convolution is law-invariant (see Example 10). This result generalizes the existing results in the literature on convex risk measures and distortion risk measures, which are all monetary. Furthermore, in all our examples in Section 6, we only require some continuity on one of the risk functionals, rather than on all of them as typically assumed in the literature.

\section{Law-invariance and inf-convolution}

Let $(\Omega, \mathcal{F}, \mathbb{P})$ be a probability space and $\mathcal{X}$ be the set of bounded random variables on this probability space. Throughout the paper, except for the motivating example, Example 1, we assume $(\Omega, \mathcal{F}, \mathbb{P})$ to be an atomless probability space, following the standard setup in the mathematical finance and risk management literature. Recall that a probability space $(\Omega, \mathcal{F}, \mathbb{P})$ is atomless if for all $B \in \mathcal{F}$ with $\mathbb{P}(B)>0$, there exists $A \in \mathcal{F}$ with $A \subset B$ such that $0<\mathbb{P}(A)<\mathbb{P}(B)$. Equivalently, there exists a continuously distributed random variable defined on this probability space. For a random variable $X$, we write $X \sim F$ if $X$ has the distribution function $F$. We use $F_{X}$ for the cdf of a random variable $X$ and $F_{X}^{-1}$ for its left quantile function, defined as $F_{X}^{-1}(t)=\inf \left\{x \in \mathbb{R}: F_{X}(x) \geqslant t\right\}, t \in(0,1]$ and $F_{X}^{-1}(0)=\inf \left\{x \in \mathbb{R}: F_{X}(x)>0\right\}=\operatorname{ess-inf}(X)$.

A risk functional is a mapping from $\mathcal{X}$ to $[-\infty, \infty) .{ }^{2}$ A risk functional $\rho$ is law-invariant if for

\footnotetext{
${ }^{2}$ It is common to require $\rho(X)<\infty$ for a bounded risk $X$. We include $-\infty$ in the range because the inf-convolution of finite functionals may be $-\infty$, e.g. the case of two VaRs (see Example 4). Therefore, to discuss properties of an
} 
all $X, Y \in \mathcal{X}$,

$$
X \stackrel{\mathrm{d}}{=} Y \quad \Rightarrow \quad \rho(X)=\rho(Y)
$$

where $\stackrel{\mathrm{d}}{=}$ stands for equality in distribution. We say that $\rho$ is law-invariant on $\mathcal{Y} \subset \mathcal{X}$ if (1) holds for all $X, Y \in \mathcal{Y}$.

For a random variable $X \in \mathcal{X}$, define the set of allocations of $X$ as

$$
\mathbb{A}_{n}(X)=\left\{\left(X_{1}, \ldots, X_{n}\right) \in \mathcal{X}^{n}: \sum_{i=1}^{n} X_{i}=X\right\} .
$$

The inf-convolution of risk functionals $\rho_{1}, \ldots, \rho_{n}$ is the mapping $\square_{i=1}^{n} \rho_{i}: \mathcal{X} \rightarrow[-\infty, \infty)$, defined as

$$
\prod_{i=1}^{n} \rho_{i}(X)=\inf \left\{\sum_{i=1}^{n} \rho_{i}\left(X_{i}\right):\left(X_{1}, \ldots, X_{n}\right) \in \mathbb{A}_{n}(X)\right\} .
$$

In particular, the inf-convolution of two risk functionals $\rho_{1}$ and $\rho_{2}$ is

$$
\rho_{1} \square \rho_{2}(X)=\inf \left\{\rho_{1}(Y)+\rho_{2}(X-Y): Y \in \mathcal{X}\right\}, \quad X \in \mathcal{X} .
$$

The concept of inf-convolution is closely related to many problems in risk sharing and general equilibrium. For instance, in general equilibrium theory, to obtain all Pareto-optimal allocations (see e.g. Starr (2011, Chapter 19)), one often needs to solve

$$
\sup \left\{\sum_{i=1}^{n} \lambda_{i} u_{i}\left(X_{i}\right):\left(X_{1}, \ldots, X_{n}\right) \in \mathbb{A}_{n}(X)\right\}
$$

for some risk $X \in \mathcal{X}$, utility functionals $u_{1}, \ldots, u_{n}: \mathcal{X} \rightarrow \mathbb{R}$ and constants $\lambda_{1}, \ldots, \lambda_{n} \geqslant 0$. Clearly, by taking $\rho_{i}=-\lambda_{i} u_{i}, i=1, \ldots, n,(5)$ becomes a special case of (3). Moreover, if $\rho_{1}, \ldots, \rho_{n}$ are monetary risk measures ${ }^{3}$ then the solutions to (3) are precisely all the Pareto-optimal allocations; see e.g. Barrieu and El Karoui (2005) and Jouini et al. (2008) for the case of convex functionals, and Embrechts et al. (2018) for the non-convex case.

In this paper, agents prefer a smaller value of their preference functional over a larger value (e.g. the case of risk measures or disutilities), and that is why we call them risk functionals. Precisely, if the binary relation $\prec$ on $\mathcal{X}$ represents the preference of an agent, and $\rho$ is its (negative) numerical representation, then

$$
X \prec Y \quad \Leftrightarrow \quad \rho(X) \geqslant \rho(Y), \quad X, Y \in \mathcal{X} .
$$

inf-convolution (as a risk functional), one has to allow the range of a risk functional to include $-\infty$.

${ }^{3}$ A functional $\rho: \mathcal{X} \rightarrow \mathbb{R}$ is a monetary risk measure if it satisfies (i) monotonicity: $\rho(X) \leqslant \rho(Y)$ for all $X, Y \in \mathcal{X}$ with $X \leqslant Y$ and (ii) cash-additivity: $\rho(X+c)=\rho(X)+c$ for all $X \in \mathcal{X}$ and $c \in \mathbb{R}$. 
In the alternative setup where agents maximize utility functionals, we can simply put a negative sign in front of $\rho$, and switch from inf-convolution to max-convolution; see e.g. (5) above. Obviously, these two settings are mathematically equivalent.

The main question of this paper is whether $\square_{i=1}^{n} \rho_{i}$ is law-invariant, if each of $\rho_{1}, \ldots, \rho_{n}$ is law-invariant. Below we collect some useful properties of a risk functional $\rho$.

(i) $\rho$ is monotone if $\rho(X) \leqslant \rho(Y)$ for all $X, Y \in \mathcal{X}$ with $X \leqslant Y$.

(ii) $\rho$ is $\preceq_{\mathrm{cx}}$-monotone, if $\rho(X) \leqslant \rho(Y)$ for all $X, Y \in \mathcal{X}$ with $X \preceq_{\mathrm{cx}} Y$, where $X \preceq_{\mathrm{cx}} Y$ means that $X \in \mathcal{X}$ is smaller than $Y \in \mathcal{X}$ in convex order, i.e. $\mathbb{E}[f(X)] \leqslant \mathbb{E}[f(Y)]$ for all convex functions $f: \mathbb{R} \rightarrow \mathbb{R}$.

(iii) Continuity of $\rho$ is defined with respect to sup-norm (with respect to $\mathbb{P}$ ), i.e. $\|X\|=\operatorname{ess}-\sup (|X|)$ for $X \in \mathcal{X}$. Recall that $\rho$ is uniformly continuous if for all $\varepsilon>0$ there exists $\delta>0$ such that for all $X, Y \in \mathcal{X},\|X-Y\| \leqslant \delta$ implies $|\rho(X)-\rho(Y)| \leqslant \varepsilon$ with the convention $(-\infty)-(-\infty)=0$.

(iv) $\rho$ is continuous from above if $X_{n} \downarrow X$ point-wise implies $\rho\left(X_{n}\right) \rightarrow \rho(X)$, and it is continuous from below if $X_{n} \uparrow X$ point-wise implies $\rho\left(X_{n}\right) \rightarrow \rho(X)$.

Commonly used risk functionals are monotone and uniformly continuous. In fact, all monetary risk measures are monotone and uniformly continuous. All law-invariant convex risk measures in the sense of Föllmer and Schied (2016) on $\mathcal{X}$ are $\preceq_{\mathrm{cx}}$-monotone.

A popular risk measure that will appear in some of our examples is the Value-at-Risk (VaR), which is the most popular risk measure used in banking and insurance, such as in Basel III/IV and Solvency II (see e.g. Embrechts et al. (2014)). We define the VaR at confidence level $\alpha \in(0,1)$ as the right $(1-\alpha)$-quantile of $X$, namely

$$
\operatorname{VaR}_{\alpha}(X)=\inf \left\{x \in \mathbb{R}: F_{X}(x)>1-\alpha\right\}, \quad X \in \mathcal{X}
$$

It is well known that for $\alpha \in(0,1), \mathrm{VaR}_{\alpha}$ is law-invariant, monotone, and uniformly continuous, but it is not $\preceq_{\mathrm{cx}}$-monotone.

\section{Motivating (counter-)examples}

\subsection{A counter-example in a finite probability space}

We first present a motivating example explaining why law-invariance may fail to hold for the inf-convolution, and we discuss its economic implication. For a better interpretation, this example 
is constructed for a finite probability space, different from the setting in the rest of the paper.

Example 1 (Counter-example in a finite probability space). Define a probability space $(\Omega, \mathcal{F}, \mathbb{P})$ where $\Omega=\left\{\omega_{1}, \omega_{2}, \omega_{3}\right\}, \mathcal{F}$ is the power set of $\Omega$, and $\mathbb{P}$ is such that $\mathbb{P}\left(\left\{\omega_{1}\right\}\right)=\mathbb{P}\left(\left\{\omega_{2}\right\}\right)=\frac{1}{4}$, $\mathbb{P}\left(\left\{\omega_{3}\right\}\right)=\frac{1}{2}$. Suppose that two agents have the risk functionals given by

$$
\rho_{1}(X)=\rho_{2}(X)=\left(\operatorname{VaR}_{1 / 3}(X)\right)_{+}, \quad X \in \mathcal{X} .
$$

That means, each agent has a disutility which is the $2 / 3$-quantile of the loss, and the minimal disutility for the agent is 0 . Such agents are similar to the quantile-based agents in Embrechts et al. (2018). Clearly, the risk functionals $\rho_{1}$ and $\rho_{2}$ are law-invariant.

Define two random variables $X=\mathbb{1}_{\left\{\omega_{1}, \omega_{2}\right\}}$, and $Y=\mathbb{1}_{\left\{\omega_{3}\right\}}$. Clearly, $X \stackrel{\mathrm{d}}{=} Y$. It is easy to see that $\rho_{1}\left(\mathbb{1}_{\left\{\omega_{1}\right\}}\right)=\operatorname{VaR}_{1 / 3}\left(\mathbb{1}_{\left\{\omega_{1}\right\}}\right)=0$ and $\rho_{2}\left(\mathbb{1}_{\left\{\omega_{2}\right\}}\right)=\operatorname{VaR}_{1 / 3}\left(\mathbb{1}_{\left\{\omega_{2}\right\}}\right)=0$, and hence $\rho_{1} \square \rho_{2}(X)=0$ noting that $\rho_{1}$ and $\rho_{2}$ are non-negative.

On the other hand, write $Y=Z+W$ for some $Z, W \in \mathcal{X}$. Set $z=Z\left(\omega_{3}\right)$ and $w=W\left(\omega_{3}\right)$. Clearly, $z+w=1$. Since $\mathbb{P}(Z=z) \geqslant 1 / 2>1 / 3$, we know that $\operatorname{VaR}_{1 / 3}(Z) \geqslant z$, and similarly $\operatorname{VaR}_{1 / 3}(W) \geqslant w$. Therefore,

$$
\rho_{1}(Z)+\rho_{2}(W) \geqslant \operatorname{VaR}_{1 / 3}(Z)+\operatorname{VaR}_{1 / 3}(W) \geqslant z+w=1
$$

This shows that $\rho_{1} \square \rho_{2}(Y) \neq 0=\rho_{1} \square \rho_{2}(X)$, and thus $\rho_{1} \square \rho_{2}$ is not law-invariant.

Example 1 has an intuitive economic explanation. Both the two agents in Example 1 are quantile-based, and hence they are not risk-averse in the classic sense (see e.g. discussions in Embrechts et al. (2019)). As non-risk-averse agents, they may prefer gambling against each other over a proportional sharing arrangement of the total loss. For the total loss $X=\mathbb{1}_{\left\{\omega_{1}, \omega_{2}\right\}}$, such gambling is possible. One can decompose $X$ into two non-negative components $\mathbb{1}_{\left\{\omega_{1}\right\}}$ and $\mathbb{1}_{\left\{\omega_{2}\right\}}$; thus the two agents can bet on which event between $\left\{\omega_{1}\right\}$ and $\left\{\omega_{2}\right\}$ happens. On the other hand, for the total loss $Y=\mathbb{1}_{\left\{\omega_{3}\right\}}$, the above arrangement is not possible; the only way to decompose $Y$ into non-negative components is proportional, that is, to decompose $Y$ into $z Y$ and $(1-z) Y$ for some $z \in[0,1]$. Since the two agents prefer gambling, the proportional loss sharing arrangement $(z Y,(1-z) Y)$ is not as favourable as the arrangement $\left(\mathbb{1}_{\left\{\omega_{1}\right\}}, \mathbb{1}_{\left\{\omega_{2}\right\}}\right)$ which is only available for the total risk $X$.

The above example shows that, as compared to $Y$, the extra randomness of $X$ improves the overall quality of the risk minimization problem, and hence the resulting inf-convolution is not the same for $X$ and $Y$, although they are identically distributed. 


\subsection{A counter-example for an atomless probability space}

We next discuss the general case of an atomless probability space, which is the standard setup for risk management. In such a probability space, there are infinitely many iid random variables, and the problem of law-invariance is much more delicate. Below we construct an example in which $\rho_{1} \square \rho_{2}$ is not law-invariant although $\rho_{1}$ and $\rho_{2}$ are both law-invariant, although this example appears to be less intuitive than Example 1.

Example 2 (Counter-example in an atomless probability space). Take $(\Omega, \mathcal{F}, \mathbb{P})=([0,1], \mathcal{B}([0,1]), \lambda)$ where $\lambda$ is the Lebesgue measure. Define three distributions $F_{B}=\operatorname{Bernoulli}\left(\frac{1}{2}\right), F_{U}=\mathrm{U}[-1,1]$, and $F=\mathrm{U}[0,1]$, and two risk measures

$$
\rho_{1}(X)=1-\mathbb{1}_{\left\{X \sim F_{B}\right\}} \text { and } \rho_{2}(X)=1-\mathbb{1}_{\left\{X \sim F_{U}\right\}}, \quad X \in \mathcal{X} .
$$

Obviously, $\rho_{1}$ and $\rho_{2}$ are law-invariant risk measures. We will show that there are random variables $X \sim F$ and $Z \sim F$ such that $\rho_{1} \square \rho_{2}(X)<\rho_{1} \square \rho_{2}(Z)$.

Since the probability space is atomless, there exist two independent random variables $X$ and $Y$ such that $X \sim F$ and $Y \sim F_{B}$. Note that $X-Y \sim F_{U}$. By (4), it is easy to see that

$$
\rho_{1} \square \rho_{2}(X) \leqslant \rho_{1}(Y)+\rho_{2}(X-Y)=0 .
$$

Hence, $\rho_{1} \square \rho_{2}(X)=0$ since $\rho_{1}$ and $\rho_{2}$ are non-negative. Next, let $Z(\omega)=\omega, \omega \in[0,1]$. It is clear that $Z \sim F$. We will show that for any $W \sim F_{B}$, the distribution of $Z-W$ cannot be $F_{U}$. Suppose for the purpose of contradiction that $Z-W \sim F_{U}$ for some $W \sim F_{B}$. For $0 \leqslant x \leqslant 1$, since $Z-W \sim F_{U}$ and $\{Z-1 \leqslant x\}=\Omega$, we have

$$
\begin{aligned}
\frac{1}{2}+\frac{x}{2} & =\mathbb{P}(Z-W \leqslant x) \\
& =\mathbb{P}(Z-W \leqslant x, W=0)+\mathbb{P}(Z-W \leqslant x, W=1) \\
& =\frac{1}{2}+\mathbb{P}(Z \leqslant x, W=0) \\
& =\frac{1}{2}+\lambda([0, x] \cap A)
\end{aligned}
$$

where $A=\{W=0\} \subset[0,1]$, which satisfies $\lambda(A)=\frac{1}{2}$. Hence

$$
\lambda([0, x] \cap A)=\frac{x}{2}, \quad 0 \leqslant x \leqslant 1 .
$$

Define a probability measure on $([0,1], \mathcal{B}([0,1]))$ by

$$
\lambda_{A}(B)=2 \lambda(B \cap A) .
$$


It follows form (7) that

$$
\lambda_{A}([0, x])=\lambda([0, x]), \quad 0 \leqslant x \leqslant 1 .
$$

By Theorem 3.3 in Billingsley (1995), two measures that agree on $\{[0, x]: x \in[0,1]\}$ also agree on $\mathcal{B}([0,1])$. Therefore, $\lambda_{A}=\lambda$. Note that $\lambda(A)=\frac{1}{2}$, and by definition $\lambda_{A}(A)=2 \lambda(A)=1$, contradicting $\lambda_{A}=\lambda$. Therefore, there does not exist $W \sim F_{B}$ such that $Z-W \sim F_{U}$. As a consequence,

$$
\rho_{1} \square \rho_{2}(Z)=1 \neq \rho_{1} \square \rho_{2}(X) .
$$

In other words, $\rho_{1} \square \rho_{2}$ is not law-invariant.

The two risk functionals $\rho_{1}$ and $\rho_{2}$ in Example 2 are rather artificial. They are not monotone or continuous, and hence they are not suitable for typical decision models. Nevertheless, we can modify them slightly, so that they are monotone and continuous from below, and after the modification the inf-convolution is still not law-invariant; see Example 15 in Section 6.

Remark 1. As a direct consequence of Example 2, for given distributions $F_{1}, \ldots, F_{n}$, the aggregation set of Bernard et al. (2014),

$$
\mathcal{S}_{n}=\left\{X_{1}+\cdots+X_{n}: X_{i} \sim F_{i}, i=1, \ldots, n\right\}
$$

is not necessarily law-invariant, i.e. $S \in \mathcal{S}_{n}$ does not necessarily imply $T \in \mathcal{S}_{n}$ for identically distributed risks $S$ and $T$. The aggregation set $\mathcal{S}_{n}$ represents all possible aggregate models with specified marginal distributions, and it is a key concept in the study of risk aggregation with dependence uncertainty; see Embrechts et al. (2013, 2015).

\subsection{Affirmative examples}

In Examples 1 and 2, we have seen that the inf-convolution of law-invariant risk functionals is not necessarily law-invariant. Nevertheless, in almost all cases studied in the literature, we observe that the inf-convolution is law-invariant. Below we give some popular examples. First, if all of $\rho_{1}, \ldots, \rho_{n}$ are $\preceq_{\mathrm{cx}}$-monotone, then their inf-convolution is law-invariant.

Example 3 ( $\preceq_{\mathrm{cx}}$-monotone functionals). We claim that $\square_{i=1}^{n} \rho_{i}$ is law-invariant if $\rho_{1}, \ldots, \rho_{n}$ are $\preceq_{\mathrm{cx}}$-monotone. To show this assertion, we rely on the comonotonic improvement (Theorems 10.47 and 10.52 of Rüschendorf (2013)), which suggests that for all $X \in \mathcal{X}$, there exists a comonotonic optimal allocation $\left(X_{1}^{*}, \ldots, X_{n}^{*}\right)$ minimizing $\sum_{i=1}^{n} \rho_{i}\left(X_{i}\right)$ over $\left(X_{1}, \ldots, X_{n}\right) \in \mathbb{A}_{n}(X)$. By Denneberg's lemma (Proposition 4.5 of Denneberg (1994)), $X_{1}^{*}, \ldots, X_{n}^{*}$ are increasing functions of $X$, and we 
denote this by $\left(X_{1}^{*}, \ldots, X_{n}^{*}\right)=\left(f_{1}(X), \ldots, f_{n}(X)\right)$. For any random variable $Y$ with $X \stackrel{\text { d }}{=} Y$, we have

$$
\square_{i=1}^{n} \rho_{i}(Y) \leqslant \sum_{i=1}^{n} \rho_{i}\left(f_{i}(Y)\right)=\sum_{i=1}^{n} \rho_{i}\left(f_{i}(X)\right)=\prod_{i=1}^{n} \rho_{i}(X) .
$$

By symmetry, $\square_{i=1}^{n} \rho_{i}$ is law-invariant.

All law-invariant quasi-convex risk functionals which are continuous with respect to the supnorm are $\preceq_{\mathrm{cx}}$-monotone (see e.g. Delbaen (2012)). As a special case, Example 3 suggests that the inf-convolution of law-invariant convex risk measures in the sense of Föllmer and Schied (2016) is law-invariant. This particular case is known in the literature of cash-additive risk measures; ${ }^{4}$ see Mao and Wang (2016, Theorem 4.1), Filipović and Svindland (2008, Corollary 2.7) and Chen et al. (2018, Proposition 3.1). Note that the assertion in Example 3 does not require quasi-convexity or cash-additivity, which are assumed in the literature.

Next, we see that without $\preceq_{\mathrm{cx}}$-monotonicity, the inf-convolution can still be law-invariant, as shown in the following two examples.

Example 4 (Inf-convolution of VaRs). By Corollary 2 of Embrechts et al. (2018), for $\alpha, \beta \in(0,1)$, if $\alpha+\beta<1$, it holds that

$$
\operatorname{VaR}_{\alpha} \square \operatorname{VaR}_{\beta}=\operatorname{VaR}_{\alpha+\beta}
$$

Here, the inf-convolution $\operatorname{VaR}_{\alpha} \square \operatorname{VaR}_{\beta}$ is law-invariant, although $\operatorname{VaR}_{\alpha}$ and $\operatorname{VaR}_{\beta}$ are not quasiconvex or $\preceq_{\mathrm{cx}}$-monotone. If $\alpha+\beta \geqslant 1$, then using Corollary 2 of Embrechts et al. (2018), we have

$$
\operatorname{VaR}_{\alpha} \square \operatorname{VaR}_{\beta}(X)=-\infty \text { for all } X \in \mathcal{X}
$$

In this case, $\operatorname{VaR}_{\alpha} \square \operatorname{VaR}_{\beta}$ is also (trivially) law-invariant. The inf-convolution of more than two VaRs is similar.

Example 5 (Inf-convolution of a VaR and a distortion risk measure). Example 4 can be generalized to include a broader class of non-convex risk measures. Let $\mathcal{G}$ be the set of non-decreasing functions $g$ on $[0,1]$ with $g(0)=0$ and $g(1)=1$. A distortion risk measure $\rho_{g}$ on $\mathcal{X}$ with distortion function $g \in \mathcal{G}$ is defined as the Choquet integral

$$
\rho_{g}(X)=\int X \mathrm{~d}(g \circ \mathbb{P})=\int_{-\infty}^{0}(g \circ \mathbb{P}(X>x)-1) \mathrm{d} x+\int_{0}^{\infty} g \circ \mathbb{P}(X>x) \mathrm{d} x, \quad X \in \mathcal{X} .
$$

\footnotetext{
${ }^{4} \rho$ is cash-additive if $\rho(X+m)=\rho(X)+m$ for all $X \in \mathcal{X}$ and $m \in \mathbb{R}$.
} 
Theorem 5.3 of Wang and Wei (2018) suggests that for $g \in \mathcal{G}$ and $\alpha \in(0,1)$,

$$
\operatorname{VaR}_{\alpha} \square \rho_{g}=\left\{\begin{array}{cl}
\rho_{g_{\alpha}} & \text { if } g(1-\alpha)=1, \\
-\infty & \text { if } g(1-\alpha)<1,
\end{array}\right.
$$

where $g_{\alpha}(t)=g\left((t-\alpha)_{+}\right), t \in[0,1]$. Here, the inf-convolution $\operatorname{VaR}_{\alpha} \square \rho_{g}$ is again law-invariant.

The above examples suggest that for commonly used law-invariant risk functionals, their infconvolution is often law-invariant. It remains unclear to what generality the above statement holds, and we focus on deriving some sufficient conditions in the rest of the paper with minimal assumptions on the form of the risk functionals.

\section{Properties of inf-convolution}

As an important tool in convex analysis, properties of inf-convolution are extensively studied for convex or quasi-convex functionals (e.g. Barrieu and El Karoui (2005), Jouini et al. (2008), Delbaen (2012)) in the risk measure literature. Inf-convolution of non-convex risk functionals such as the VaR is studied recently by Embrechts et al. (2018, 2019), Weber (2018) and Wang and Wei (2018). To establish results with minimal assumptions on the risk functionals, we need some simple properties of inf-convolution without the conditions assumed in the literature. These properties will be useful for the main results of this paper in Section 5.

First, we show that some of the properties in Section 2 can be naturally passed on from $\rho_{1}$ and $\rho_{2}$ to $\rho_{1} \square \rho_{2}$.

Lemma 1. Let $\rho_{1}$ and $\rho_{2}$ be two risk functionals.

(i) If $\rho_{1}$ or $\rho_{2}$ is monotone, then so is $\rho_{1} \square \rho_{2}$.

(ii) If $\rho_{1}$ or $\rho_{2}$ is uniformly continuous, then so is $\rho_{1} \square \rho_{2}$.

(iii) If $\rho_{1}$ or $\rho_{2}$ is monotone and continuous from above, then so is $\rho_{1} \square \rho_{2}$.

Proof. Without loss of generality, we assume in each case of (i)-(iii), $\rho_{2}$ has the specified property.

(i) For $X, Y \in \mathcal{X}$ with $X \leqslant Y$, let $\delta=Y-X \geqslant 0$. By (4), we have

$$
\begin{aligned}
\rho_{1} \square \rho_{2}(Y)=\rho_{1} \square \rho_{2}(X+\delta) & =\inf \left\{\rho_{1}(Z)+\rho_{2}(X+\delta-Z): Z \in \mathcal{X}\right\} \\
& \geqslant \inf \left\{\rho_{1}(Z)+\rho_{2}(X-Z): Z \in \mathcal{X}\right\}=\rho_{1} \square \rho_{2}(X) .
\end{aligned}
$$

Hence, $\rho_{1} \square \rho_{2}$ is monotone. 
(ii) Since $\rho_{2}$ is uniformly continuous, for any $\varepsilon>0$, there exists $\delta>0$ such that for all $X, Y \in \mathcal{X}$, $\|X-Y\| \leqslant \delta$ implies $\left|\rho_{2}(X)-\rho_{2}(Y)\right| \leqslant \varepsilon$. For any $X, Y \in \mathcal{X}$ with $\|X-Y\| \leqslant \delta$, by (4), we have

$$
\begin{aligned}
& \left|\rho_{1} \square \rho_{2}(Y)-\rho_{1} \square \rho_{2}(X)\right| \\
& =\left|\inf \left\{\rho_{1}(Z)+\rho_{2}(Y-Z): Z \in \mathcal{X}\right\}-\inf \left\{\rho_{1}(Z)+\rho_{2}(X-Z): Z \in \mathcal{X}\right\}\right| \\
& \leqslant \sup \left\{\left|\left(\rho_{1}(Z)+\rho_{2}(Y-Z)\right)-\left(\rho_{1}(Z)+\rho_{2}(X-Z)\right)\right|: Z \in \mathcal{X}\right\} \\
& =\sup \left\{\left|\rho_{2}(Y-Z)-\rho_{2}(X-Z)\right|: Z \in \mathcal{X}\right\} \leqslant \varepsilon .
\end{aligned}
$$

Hence, $\rho_{1} \square \rho_{2}$ is uniformly continuous.

(iii) By part (i), we know that $\rho_{1} \square \rho_{2}$ is monotone. Let $\left\{X_{n}\right\}_{n \in \mathbb{N}}$ be a sequence in $\mathcal{X}$ such that $X_{n} \downarrow X$ as $n \rightarrow \infty$. We can calculate

$$
\begin{aligned}
\limsup _{n \rightarrow \infty} \rho_{1} \square \rho_{2}\left(X_{n}\right) & =\limsup _{n \rightarrow \infty}\left(\inf \left\{\rho_{1}(Z)+\rho_{2}\left(X_{n}-Z\right): Z \in \mathcal{X}\right\}\right) \\
& \leqslant \inf \left\{\rho_{1}(Z)+\rho_{2}(X-Z): Z \in \mathcal{X}\right\}=\rho_{1} \square \rho_{2}(X) .
\end{aligned}
$$

On the other hand, since $\rho_{1} \square \rho_{2}$ is monotone, we have $\rho_{1} \square \rho_{2}\left(X_{n}\right) \geqslant \rho_{1} \square \rho_{2}(X)$. This implies

$$
\lim _{n \rightarrow \infty} \rho_{1} \square \rho_{2}\left(X_{n}\right)=\rho_{1} \square \rho_{2}(X) .
$$

Hence, $\rho_{1} \square \rho_{2}$ is continuous from above.

Unlike uniform continuity or monotonicity in Lemma 1, continuity cannot be passed from $\rho_{1}$ and $\rho_{2}$, as illustrated in the following example.

Example 6 ( $\rho_{1} \square \rho_{2}$ is not continuous for continuous $\rho_{1}$ and $\rho_{2}$ ). Let $\rho_{1}(X)=\mathbb{E}\left[X^{2}\right]=-\rho_{2}(X), X \in$ $\mathcal{X}$. Clearly, both $\rho_{1}$ and $\rho_{2}$ are continuous. We can calculate

$$
\rho_{1} \square \rho_{2}(0)=\inf \left\{\rho_{1}(Y)+\rho_{2}(-Y): Y \in \mathcal{X}\right\}=0,
$$

and for $\varepsilon \neq 0$,

$$
\begin{aligned}
\rho_{1} \square \rho_{2}(\varepsilon)=\inf \left\{\rho_{1}(Y+\varepsilon)+\rho_{2}(-Y): Y \in \mathcal{X}\right\} & \leqslant \inf \left\{(y+\varepsilon)^{2}-y^{2}: y \in \mathbb{R}\right\} \\
& =\inf \left\{2 y \varepsilon+\varepsilon^{2}: y \in \mathbb{R}\right\}=-\infty
\end{aligned}
$$

Therefore, $\rho_{1} \square \rho_{2}$ is not continuous at 0 . 
The next lemma justifies the simple facts that the inf-convolution of two risk functionals is exchangeable, and the inf-convolution of $n$ risk functionals can be seen as the repeated application of inf-convolutions of two risk functionals. In the expression $\rho_{1} \square \rho_{2} \square \ldots \square \rho_{n}$ below, the convention is to perform the operations $\square$ from left to right.

Lemma 2. For any risk functionals $\rho_{1}, \ldots, \rho_{n}$, we have $\rho_{1} \square \rho_{2}=\rho_{2} \square \rho_{1}$ and

$$
\prod_{i=1}^{n} \rho_{i}=\rho_{1} \square \rho_{2} \square \ldots \square \rho_{n} .
$$

Proof. The exchangeability $\rho_{1} \square \rho_{2}=\rho_{2} \square \rho_{1}$ follows directly from the formulation (4). To show (8), we use induction. The statement (8) trivially holds for $n=2$. Next, suppose that $\square_{i=1}^{k} \rho_{i}=$ $\rho_{1} \square \rho_{2} \square \ldots \square \rho_{k}$ holds for $k \leqslant n-1$. For $X \in \mathcal{X}$ and any allocation $\left(X_{1}, \ldots, X_{n}\right) \in \mathbb{A}_{n}(X)$, we have

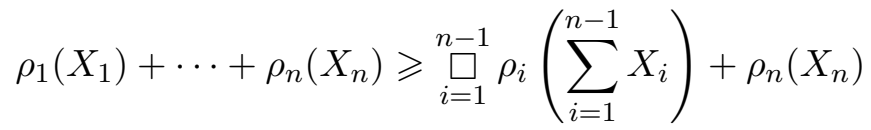

$$
\begin{aligned}
& =\rho_{1} \square \rho_{2} \square \ldots \square \rho_{n-1}\left(\sum_{i=1}^{n-1} X_{i}\right)+\rho_{n}\left(X_{n}\right) \\
& \geqslant\left(\rho_{1} \square \ldots \square \rho_{n-1}\right) \square \rho_{n}(X) .
\end{aligned}
$$

Hence, $\square_{i=1}^{n} \rho_{i} \geqslant \rho_{1} \square \rho_{2} \square \ldots \square \rho_{n}$. To show the converse direction, fix $\varepsilon>0$. By definition of inf-convolution, there exists $\left(Y, X_{n}\right) \in \mathbb{A}_{2}(X)$ be such that

$$
\rho_{1} \square \rho_{2} \square \ldots \square \rho_{n-1}(Y)+\rho_{n}\left(X_{n}\right) \leqslant \rho_{1} \square \rho_{2} \square \ldots \square \rho_{n}(X)+\frac{\varepsilon}{2} .
$$

Similarly, there exists $\left(X_{1}, \ldots, X_{n-1}\right) \in \mathbb{A}_{n-1}(Y)$ such that

$$
\rho_{1}\left(X_{1}\right)+\cdots+\rho_{n-1}\left(X_{n-1}\right) \leqslant \stackrel{n-1}{\square} \rho_{i}(Y)+\frac{\varepsilon}{2}=\rho_{1} \square \rho_{2} \square \ldots \square \rho_{n-1}(Y)+\frac{\varepsilon}{2} .
$$

Putting (9) and (10) together, there exists $\left(X_{1}, \ldots, X_{n}\right) \in \mathbb{A}_{n}(X)$ such that

$$
\rho_{1}\left(X_{1}\right)+\cdots+\rho_{n}\left(X_{n}\right) \leqslant \rho_{1} \square \rho_{2} \square \ldots \square \rho_{n}(X)+\varepsilon
$$

Since $\varepsilon>0$ is arbitrary, we obtain (8). The proof is complete by induction over $n \in \mathbb{N}$.

Remark 2. As a direct consequence of Lemma 2, the operation $\square$ is both commutative and associative. 


\section{$5 \quad$ Sufficient conditions for law-invariance}

In this section, we provide three conditions under which law-invariance is preserved under infconvolution. The three conditions are: (i) the underlying probability space is very rich; (ii) one of the risk functionals is continuous; (iii) one of the risk functionals is monotone and continuous from above. Among these conditions, (i) is not very realistic as the probability space will be extremely rich; nevertheless, (i) assumes nothing on the risk functionals but only the underlying probability space. On the other hand, conditions (ii)-(iii) are very weak and they are satisfied by commonly used risk functionals; see Section 6 for examples. The main results are summarized in Theorems 1 and 2.

\subsection{A sufficient condition on the underlying probability space}

We start this section by showing that if there is sufficient sources of randomness for constructing the allocation in $\mathbb{A}_{n}(X)$, then the inf-convolution preserves law-invariance. For this purpose, define the following set

$$
\mathcal{X}^{\perp}=\{X \in \mathcal{X}: \text { there exists a } \mathrm{U}[0,1] \text { random variable independent of } X\} .
$$

Theorem 1. If $\rho_{1}, \ldots, \rho_{n}$ are law-invariant risk functionals, then $\square_{i=1}^{n} \rho_{i}$ is law-invariant on $\mathcal{X}^{\perp}$. In particular, if $\mathcal{X}=\mathcal{X}^{\perp}$, then $\square_{i=1}^{n} \rho_{i}$ is law-invariant.

Proof. Take $X, Y \in \mathcal{X}^{\perp}$ with $X \stackrel{\mathrm{d}}{=} Y$ and an arbitrary $\left(X_{1}, \ldots, X_{n}\right) \in \mathbb{A}_{n}(X)$. Since $Y \in \mathcal{X}^{\perp}$, there exist iid $\mathrm{U}[0,1]$ random variables $U_{1}, \ldots, U_{n}$ independent of $Y$, noting that one can easily generate a (countable) sequence of iid random variables from one uniform random variable (see e.g. Theorem 1 of Delbaen (2012)).

Let $Y_{1}=F_{X_{1} \mid X}^{-1}\left(U_{1} \mid Y\right)$ where $F_{X_{1} \mid X}^{-1}(\cdot \mid y)$ is the quantile function of $X_{1}$ given $X=y \in \mathbb{R}$. It is easy to check $\left(Y, Y_{1}\right) \stackrel{\mathrm{d}}{=}\left(X, X_{1}\right)$. Repeating the above procedure, for $j=2, \ldots, n$, let

$$
Y_{j}=F_{X_{j} \mid X_{j-1}, \ldots, X_{1}, X}^{-1}\left(U_{j} \mid Y_{j-1}, \ldots, Y_{1}, Y\right)
$$

where $F_{X_{j} \mid X_{j-1}, \ldots, X_{1}, X}^{-1}\left(\cdot \mid y_{j-1}, \ldots, y_{1}, y\right)$ is the quantile function of $X_{j}$ given $\left(X_{j-1}, \ldots, X_{1}, X\right)=$ $\left(y_{j-1}, \ldots, y_{1}, y\right) \in \mathbb{R}^{j}$. By construction, we have $\left(Y, Y_{1}, \ldots, Y_{n}\right) \stackrel{\mathrm{d}}{=}\left(X, X_{1}, \ldots, X_{n}\right)$, and as a consequence, $\left(Y_{1}, \ldots, Y_{n}\right) \in \mathbb{A}_{n}(Y)$. Hence,

$$
\square_{i=1}^{n} \rho_{i}(Y) \leqslant \sum_{i=1}^{n} \rho_{i}\left(Y_{i}\right)=\sum_{i=1}^{n} \rho_{i}\left(X_{i}\right) .
$$


Taking an infimum over $\left(X_{1}, \ldots, X_{n}\right) \in \mathbb{A}_{n}(X)$ in the above equation, we obtain $\square_{i=1}^{n} \rho_{i}(Y) \leqslant$ $\square_{i=1}^{n} \rho_{i}(X)$. By symmetry, $\square_{i=1}^{n} \rho_{i}(Y)=\square_{i=1}^{n} \rho_{i}(X)$.

By Theorem $1, \mathcal{X}^{\perp}=\mathcal{X}$ is a sufficient condition for law-invariance to be preserved by infconvolution. The next few examples discuss properties related to the set $\mathcal{X}^{\perp}$, and in particular, whether $\mathcal{X}^{\perp}=\mathcal{X}$. First, we see that typically $\mathcal{X}^{\perp}$ is a subset of $\mathcal{X}$ which does not coincide with $\mathcal{X}$.

Example $7\left(\mathcal{X}^{\perp} \neq \mathcal{X}\right)$. Suppose that $(\Omega, \mathcal{F}, \mathbb{P})=([0,1], \mathcal{B}([0,1]), \lambda)$ where $\lambda$ is the Lebesgue measure. Define the random variable $X \in \mathcal{X}$ by the identity mapping $X(\omega)=\omega, \omega \in[0,1]$. Since there is no non-degenerate random variable in $\mathcal{X}$ independent of $X$, we know that $X \notin \mathcal{X}^{\perp}$. Hence $\mathcal{X}^{\perp} \subsetneq \mathcal{X}$

Probability spaces that are isomorphic to $([0,1], \mathcal{B}([0,1]), \lambda)$ in Example 7 are called standard probability spaces, and they are the typical choice in risk management (see e.g. Delbaen (2002) and Jouini et al. (2006)). A standard probability space allows for the existence of countably many Brownian motions, and thus it is enough for most relevant problems in the field. Note that all standard probability spaces lead to $\mathcal{X}^{\perp} \neq \mathcal{X}$ due to their isomorphism to $([0,1], \mathcal{B}([0,1]), \lambda)$.

The next example shows that $\mathcal{X}^{\perp}$ is not necessarily a convex set. This suggests that lawinvariance on $\mathcal{X}^{\perp}$ may not be a very convenient property, since it is not closed under addition or convex combination.

Example $8\left(\mathcal{X}^{\perp}\right.$ is not convex). Again, suppose that $(\Omega, \mathcal{F}, \mathbb{P})=([0,1], \mathcal{B}([0,1]), \lambda)$ where $\lambda$ is the Lebesgue measure, and $X \in \mathcal{X}$ is the identity mapping $X(\omega)=\omega, \omega \in[0,1]$. We write $X$ in its binary form

$$
X=\sum_{k \in \mathbb{N}} 2^{-k} Z_{k}
$$

where $\left\{Z_{k}\right\}_{k \in \mathbb{N}}$ is a sequence of iid Bernoulli random variables with mean $1 / 2$. Define four random variables

$$
X_{1}=\sum_{k \in \mathbb{N}} 2^{-(2 k-1)} Z_{2 k-1}, \quad X_{2}=\sum_{k \in \mathbb{N}} 2^{-2 k} Z_{2 k}
$$

and

$$
Y_{1}=\sum_{k \in \mathbb{N}} 2^{-k} Z_{2 k-1}, \quad Y_{2}=\sum_{k \in \mathbb{N}} 2^{-k} Z_{2 k}
$$

It is easy to see that both $Y_{1}$ and $Y_{2}$ are uniformly distributed over [0,1], $X_{1}, Y_{2}$ are independent, and $X_{2}, Y_{1}$ are independent. Hence, $X_{1}, X_{2} \in \mathcal{X}^{\perp}$. However, $\frac{1}{2}\left(X_{1}+X_{2}\right)=\frac{1}{2} X \notin \mathcal{X}^{\perp}$, showing that $\mathcal{X}^{\perp}$ is not convex. 
On the other hand, there exist some (non-standard) probability spaces which allow for $\mathcal{X}^{\perp}=\mathcal{X}$. Such probability spaces are very large and they involve uncountably many iid random variables, as shown in the following example.

Example $9\left(\mathcal{X}^{\perp}=\mathcal{X}\right)$. Let $(\Omega, \mathcal{F}, \mathbb{P})$ be a probability space on which a class of iid $\mathrm{N}(0,1)$ random variables $X_{t}, t \in[0,1]$ are defined. The existence of a such probability space is guaranteed by the Kolmogorov consistency Theorem (see, e.g., Theorem 2.2 in Karatzas and Shreve (1991)). For $I \subset[0,1], \mathcal{F}_{I}$ denotes the $\sigma$-algebra generated by the random variables $X_{t}, t \in I$. Let

$$
\mathcal{F}^{*}=\bigcup_{I \subset[0,1] \text { and } I \text { is countable }} \mathcal{F}_{I}
$$

Clearly, $\mathcal{F}^{*}$ is a $\sigma$-algebra and $X_{t}, t \in[0,1]$ is well defined on $\left(\Omega, \mathcal{F}^{*}, \mathbb{P}\right)$. For any random variable $Y$ on $\left(\Omega, \mathcal{F}^{*}, \mathbb{P}\right)$ and $q \in \mathbb{Q}$, where $\mathbb{Q}$ is the set of all rational numbers, due to (12), there exists a countable set $I_{q} \subset[0,1]$ such that $\{Y<q\} \in \mathcal{F}_{I_{q}}$. Denote by $J=\bigcup_{q \in \mathbb{Q}} I_{q}$. It follows that

$$
\sigma(Y)=\sigma(\{Y<q\}, q \in \mathbb{Q}) \subset \sigma\left(X_{t}, t \in \bigcup_{q \in \mathbb{Q}} I_{q}\right)=\mathcal{F}_{J} .
$$

Note that $[0,1] \backslash J \neq \emptyset$ since $J$ is countable. Then there exists $t_{0} \in[0,1] \backslash J$ such that $X_{t_{0}}$ is independent of $X_{t}, t \in J$. Therefore, $X_{t_{0}}$ is independent of $Y$ and $\Phi\left(X_{t_{0}}\right)$ is a uniform random variable where $\Phi$ is the standard normal cdf. Hence $\left(\Omega, \mathcal{F}^{*}, \mathbb{P}\right)$ satisfies $\mathcal{X}^{\perp}=\mathcal{X}$.

Below we present a useful lemma showing that discrete random variables are always elements of $\mathcal{X}^{\perp}$. This lemma will be useful to establish Theorem 2 .

Lemma 3. If $X \in \mathcal{X}$ takes values in a countable set, then $X \in \mathcal{X}^{\perp}$.

Proof. Suppose that $X$ takes values in a countable set $M$. Let $U_{X}$ be a U[0,1] random variable such that $X=F_{X}^{-1}\left(U_{X}\right)$ a.s.; such a random variable always exists (e.g. Lemma A.32 of Föllmer and Schied (2016)). Define a random variable $U$ as

$$
U=\frac{F_{X}(X)-U_{X}}{F_{X}(X)-F_{X}(X-)}
$$

where $F_{X}(x-)=\lim _{y \uparrow x} F_{X}(y)$ for $x \in \mathbb{R}$. Note that $U$ is well defined since $F_{X}(X)>F_{X}(X-)$ a.s. for the discrete random variable $X$. We can calculate, for $u \in[0,1]$, and $x \in M$,

$$
\begin{aligned}
\mathbb{P}(U \leqslant u \mid X=x) & =\mathbb{P}\left(\frac{F_{X}(x)-U_{X}}{F_{X}(x)-F_{X}(x-)} \leqslant u \mid F_{X}^{-1}\left(U_{X}\right)=x\right) \\
& =\mathbb{P}\left(\frac{F_{X}(x)-U_{X}}{F_{X}(x)-F_{X}(x-)} \leqslant u \mid F_{X}(x-)<U_{X} \leqslant F_{X}(x)\right) .
\end{aligned}
$$


Note that conditionally on $F_{X}(x-)<U_{X} \leqslant F_{X}(x), \frac{F_{X}(x)-U_{X}}{F_{X}(x)-F_{X}(x-)}$ is a $\mathrm{U}[0,1]$ random variable. Hence, $\mathbb{P}(U \leqslant u \mid X=x)=u, u \in[0,1]$. This implies that $U$ is $\mathrm{U}[0,1]$-distributed, and it is independent of $X$.

Putting Lemma 3 and Theorem 1 together, we know that if $\rho_{1}, \ldots, \rho_{n}$ are law-invariant, then $\square_{i=1}^{n} \rho_{i}(X)=\square_{i=1}^{n} \rho_{i}(Y)$ for all identically distributed discrete random variables $X$ and $Y$.

\subsection{Sufficient conditions on the risk functionals}

As we have seen from Lemma 3, law-invariance is generally preserved on discrete random variables. Inspired by this observation, for any random variable $X \in \mathcal{X}$, we define two sequences of discrete approximations $\left\{\bar{X}_{n}\right\}_{n \in \mathbb{N}}$ and $\left\{\underline{X}_{n}\right\}_{n \in \mathbb{N}}$ by

$$
\bar{X}_{n}=\frac{1}{n}\lceil n X\rceil \text { and } \underline{X}_{n}=\frac{1}{n}\lfloor n X\rfloor,
$$

where for $x \in \mathbb{R},\lceil x\rceil$ is the smallest integer greater than or equal to $x$ and $\lfloor x\rfloor$ is the greatest integer smaller than or equal to $x$. Note that $\underline{X}_{n} \leqslant X \leqslant \bar{X}_{n}$ and $\bar{X}_{n}-\underline{X}_{n} \leqslant 1 / n$. Using the above approximations, we obtain the law-invariance of $\square_{i=1}^{n} \rho_{i}$ under an assumption of continuity.

Proposition 1. If $\rho_{1}, \ldots, \rho_{n}$ are law-invariant risk functionals, and $\square_{i=1}^{n} \rho_{i}$ is either continuous from above or below in sup-norm, then $\square_{i=1}^{n} \rho_{i}$ is law-invariant.

Proof. Take $X, Y$ with $X \stackrel{\mathrm{d}}{=} Y$. Note that $\bar{X}_{n}, \underline{X}_{n}, \bar{Y}_{n}$ and $\underline{Y}_{n}$ all take finitely many values, and hence they belong to $\mathcal{X}^{\perp}$ by Lemma 3. Further, it is easy to see that $\underline{X}_{n} \stackrel{\mathrm{d}}{=} \underline{Y}_{n}$ and $\bar{X}_{n} \stackrel{\mathrm{d}}{=} \bar{Y}_{n}$. Applying Theorem 1, we obtain that for each $n \in \mathbb{N}, \square_{i=1}^{n} \rho_{i}\left(\underline{X}_{n}\right)=\square_{i=1}^{n} \rho_{i}\left(\underline{Y}_{n}\right)$ and $\square_{i=1}^{n} \rho_{i}\left(\bar{X}_{n}\right)=$ $\square_{i=1}^{n} \rho_{i}\left(\bar{Y}_{n}\right)$. Moreover, note that $\bar{X}_{2^{n}} \downarrow X$ and $\bar{Y}_{2^{n}} \downarrow Y$ in sup-norm as $n \rightarrow \infty$, and $\underline{X}_{2^{n}} \uparrow X$ and $\underline{Y}_{2^{n}} \uparrow Y$ in sup-norm as $n \rightarrow \infty$. If $\square_{i=1}^{n} \rho_{i}$ is continuous from above, we have

$$
\square_{i=1}^{n} \rho_{i}(Y)=\lim _{n \rightarrow \infty} \square_{i=1}^{n} \rho_{i}\left(\bar{Y}_{2^{n}}\right)=\lim _{n \rightarrow \infty} \square_{i=1}^{n} \rho_{i}\left(\bar{X}_{2^{n}}\right)=\square_{i=1}^{n} \rho_{i}(X) .
$$

Therefore, $\square_{i=1}^{n} \rho_{i}$ is law-invariant. If $\square_{i=1}^{n} \rho_{i}$ is continuous from below, it suffices to replace $\bar{X}_{2^{n}}$ and $\bar{Y}_{2^{n}}$ by $\underline{X}_{2^{n}}$ and $\underline{Y}_{2^{n}}$ in (13).

The continuity of $\square_{i=1}^{n} \rho_{i}$ in Proposition 1 may sometimes be inconvenient to check, if one does not know the form of $\square_{i=1}^{n} \rho_{i}$ or its properties. The following theorem, which is the main result of this paper, shows that some continuity of one of the individual law-invariant risk functionals is sufficient for the inf-convolution to be law-invariant, and this conclusion holds under three different conditions. 
Theorem 2. Suppose that $\rho_{1}, \ldots, \rho_{n}$ are law-invariant risk functionals.

(i) If one of $\rho_{1}, \ldots, \rho_{n}$ is uniformly continuous, then $\square_{i=1}^{n} \rho_{i}$ is law-invariant and uniformly continuous.

(ii) If one of $\rho_{1}, \ldots, \rho_{n}$ is monotone and continuous from above, then $\square_{i=1}^{n} \rho_{i}$ is law-invariant, monotone and continuous from above.

(iii) If one of $\rho_{1}, \ldots, \rho_{n}$ is continuous, then $\square_{i=1}^{n} \rho_{i}$ is law-invariant.

Proof. We first consider cases (i) and (ii). Without loss of generality, assume that $\rho_{1}$ has the specified properties (uniform continuity, or monotonicity and continuity from above). By Lemma 1, $\rho_{1} \square \rho_{2}$ has the specified properties, and hence it is law-invariant due to Proposition 1. Repeating the above procedure $n-1$ times, we know that $\rho_{1} \square \ldots \square \rho_{n}$ has the specified properties and is law-invariant. By Lemma 2, $\square_{i=1}^{n} \rho_{i}=\rho_{1} \square \ldots \square \rho_{n}$.

We next focus on case (iii). Without loss of generality, we assume that $\rho_{n}$ is continuous. Take $X, Y \in$ $\mathcal{X}$ with $X \stackrel{\mathrm{d}}{=} Y$. To show law-invariance of $\square_{i=1}^{n} \rho_{i}$, it suffices to show that for any $Z_{1}, \ldots, Z_{n-1} \in \mathcal{X}$, there exist sequences $\left\{W_{i, k}\right\}_{k \in \mathbb{N}} \subset \mathcal{X}, i=1, \ldots, n-1$ such that

$$
W_{i, k} \stackrel{\mathrm{d}}{=} Z_{i} \text { for all } i, k \text { and } \lim _{k \rightarrow \infty} \rho_{n}\left(Y-\sum_{i=1}^{n-1} W_{i, k}\right)=\rho_{n}\left(X-\sum_{i=1}^{n-1} Z_{i}\right) .
$$

This is because (14) implies

$$
\begin{aligned}
\square_{i=1}^{n} \rho_{i}(Y) & \leqslant \inf _{k \in \mathbb{N}}\left\{\sum_{i=1}^{n-1} \rho_{i}\left(W_{i, k}\right)+\rho_{n}\left(Y-\sum_{i=1}^{n-1} W_{i, k}\right)\right\} \\
& \leqslant \sum_{i=1}^{n-1} \rho_{i}\left(Z_{i}\right)+\rho_{n}\left(X-\sum_{i=1}^{n-1} Z_{i}\right) .
\end{aligned}
$$

Since $Z_{1}, \ldots, Z_{n-1}$ are arbitrary, this implies $\square_{i=1}^{n} \rho_{i}(Y) \leqslant \square_{i=1}^{n} \rho_{i}(X)$, and by symmetry, it is $\square_{i=1}^{n} \rho_{i}(Y)=\square_{i=1}^{n} \rho_{i}(X)$. Therefore, it is sufficient to show that for any choice of $\left(Z_{1}, \ldots, Z_{n-1}\right)$, there exist sequences $\left\{W_{i, k}\right\}_{k \in \mathbb{N}} \subset \mathcal{X}, i=1, \ldots, n-1$ such that (14) holds.

Write $Z=\sum_{i=1}^{n-1} Z_{i}$ and since $X$ and $Z$ are bounded random variables, we can assume without loss of generality that $|X|<1$ and $|Z|<1$. Fix $k \in \mathbb{N}$. Since the probability space is atomless and $X \stackrel{\mathrm{d}}{=} Y$, there exist disjoint sets $A_{\ell}, \ell \in[-k, k-1] \cap \mathbb{N}$ such that for all integers $j \in[-k, k]$,

$$
\mathbb{P}\left(\left\{Y \leqslant \frac{j}{k}\right\} \cap A_{\ell}\right)=\mathbb{P}\left(\left\{X \leqslant \frac{j}{k}\right\} \cap\left\{\frac{\ell}{k}<Z \leqslant \frac{\ell+1}{k}\right\}\right) .
$$

Note that $\mathbb{P}\left(A_{\ell}\right)=\mathbb{P}\left(\frac{\ell}{k}<Z \leqslant \frac{\ell+1}{k}\right)$ by taking $j=k$, and by Lemma $3, \mathbb{1}_{A_{\ell}} \in \mathcal{X}^{\perp}$. Hence, for $\ell \in[-k, k-1] \cap \mathbb{N}$, there exists a random vector $\left(W_{1, k}^{(\ell)}, \ldots, W_{n-1, k}^{(\ell)}\right)$ independent of $A_{\ell}$ such that

$$
\mathbb{P}\left(\left(W_{1, k}^{(\ell)}, \ldots, W_{n-1, k}^{(\ell)}\right) \leqslant \mathbf{w}\right)=\mathbb{P}\left(\left(Z_{1}, \ldots, Z_{n-1}\right) \leqslant \mathbf{w} \mid \frac{\ell}{k}<Z \leqslant \frac{\ell+1}{k}\right) \text { for all } \mathbf{w} \in \mathbb{R}^{n-1},
$$


where we set $\left(W_{1, k}^{(\ell)}, \ldots, W_{n-1, k}^{(\ell)}\right)=\mathbf{0}$ if $\mathbb{P}\left(\frac{\ell}{k}<Z \leqslant \frac{\ell+1}{k}\right)=0$. Define random variables $W_{i, k}$, $i=1, \ldots, n-1$, and $W_{k}$ by

$$
W_{i, k}=\sum_{\ell=-k}^{k-1} \mathbb{1}_{A_{\ell}} W_{i, k}^{(\ell)} \text { and } \quad W_{k}=\sum_{i=1}^{n-1} W_{i, k} .
$$

By construction, we have $W_{i, k} \stackrel{\mathrm{d}}{=} Z_{i}, i=1, \ldots, n-1$. For any $x \in \mathbb{R}$,

$$
\begin{aligned}
\mathbb{P}\left(W_{k} \leqslant x\right) & =\sum_{\ell=-k}^{k-1} \mathbb{P}\left(\sum_{i=1}^{n-1} W_{i, k} \leqslant x, A_{\ell}\right) \\
& =\sum_{\ell=-k}^{k-1} \mathbb{P}\left(\sum_{i=1}^{n-1} W_{i, k}^{(\ell)} \leqslant x, A_{\ell}\right) \\
& =\sum_{\ell=-k}^{k-1} \mathbb{P}\left(\sum_{i=1}^{n-1} Z_{i} \leqslant x, \frac{\ell}{k}<Z \leqslant \frac{\ell+1}{k}\right)=\mathbb{P}(Z \leqslant x) .
\end{aligned}
$$

Moreover, for all integers $\ell \in[-k, k-1]$,

$$
\mathbb{P}\left(\frac{\ell}{k}<W_{k} \leqslant \frac{\ell+1}{k}\right)=\mathbb{P}\left(\left\{\frac{\ell}{k}<W_{k} \leqslant \frac{\ell+1}{k}\right\} \cap A_{\ell}\right)=\mathbb{P}\left(A_{\ell}\right),
$$

which implies

$$
\left\{\frac{\ell}{k}<W_{k} \leqslant \frac{\ell+1}{k}\right\}=A_{\ell} \quad \text { a.s. }
$$

Observe that for any $x \in[j / k,(j+1) / k],-2 k \leqslant j \leqslant 2 k-1$ and $-k \leqslant \ell \leqslant k-1$,

$$
\begin{aligned}
\mathbb{P}\left(Y-W_{k} \leqslant x, \frac{\ell}{k}<W_{k} \leqslant \frac{\ell+1}{k}\right) & \leqslant \mathbb{P}\left(Y \leqslant \frac{j+\ell+2}{k}, \frac{\ell}{k}<W_{k} \leqslant \frac{\ell+1}{k}\right) \\
& =\mathbb{P}\left(X \leqslant \frac{j+\ell+2}{k}, \frac{\ell}{k}<Z \leqslant \frac{\ell+1}{k}\right) \\
& \leqslant \mathbb{P}\left(X-Z \leqslant \frac{j+2}{k}, \frac{\ell}{k}<Z \leqslant \frac{\ell+1}{k}\right) \\
& \leqslant \mathbb{P}\left(X-Z \leqslant x+\frac{2}{k}, \frac{\ell}{k}<Z \leqslant \frac{\ell+1}{k}\right) .
\end{aligned}
$$

The above inequality is obviously also valid for $|x| \geqslant 2$. Hence

$$
\mathbb{P}\left(Y-W_{k} \leqslant x, \frac{\ell}{k}<W_{k} \leqslant \frac{\ell+1}{k}\right) \leqslant \mathbb{P}\left(X-Z \leqslant x+\frac{2}{k}, \frac{\ell}{k}<Z \leqslant \frac{\ell+1}{k}\right), \quad x \in \mathbb{R} .
$$

Analogously,

$$
\mathbb{P}\left(Y-W_{k} \leqslant x, \frac{\ell}{k}<W_{k} \leqslant \frac{\ell+1}{k}\right) \geqslant \mathbb{P}\left(X-Z \leqslant x-\frac{2}{k}, \frac{\ell}{k}<Z \leqslant \frac{\ell+1}{k}\right), \quad x \in \mathbb{R} .
$$

Noting that

$$
\mathbb{P}(X-Z \leqslant x)=\sum_{\ell=-k}^{k-1} \mathbb{P}\left(X-Z \leqslant x, \frac{\ell}{k}<Z \leqslant \frac{\ell+1}{k}\right),
$$


and

$$
\mathbb{P}\left(Y-W_{k} \leqslant x\right)=\sum_{\ell=-k}^{k-1} \mathbb{P}\left(X-W_{k} \leqslant x, \frac{\ell}{k}<W_{k} \leqslant \frac{\ell+1}{k}\right),
$$

we have

$$
F_{X-Z}\left(x-\frac{2}{k}\right) \leqslant F_{Y-W_{k}}(x) \leqslant F_{X-Z}\left(x+\frac{2}{k}\right), \quad x \in \mathbb{R} .
$$

Consequently,

$$
F_{X-Z}^{-1}(t)-\frac{2}{k} \leqslant F_{Y-W_{k}}^{-1}(t) \leqslant F_{X-Z}^{-1}(t)+\frac{2}{k}, \quad t \in(0,1),
$$

which implies that, for a uniform random variable $U$ taking values on $[0,1]$,

$$
\lim _{k \rightarrow \infty} \operatorname{ess-sup}\left|F_{Y-W_{k}}^{-1}(U)-F_{X-Z}^{-1}(U)\right|=0 .
$$

By continuity and law-invariance of $\rho_{n}$, we have

$$
\lim _{k \rightarrow \infty} \rho_{n}\left(Y-W_{k}\right)=\lim _{k \rightarrow \infty} \rho_{n}\left(F_{Y-W_{k}}^{-1}(U)\right)=\rho_{n}\left(F_{X-Z}^{-1}(U)\right)=\rho_{n}(X-Z) .
$$

This completes the proof.

The conditions in Theorem 2 are very weak and satisfied by almost all commonly used risk functionals; some examples are collected in Section 6. Not that in case (iii) of Theorem $2, \square_{i=1}^{n} \rho_{i}$ is not necessarily continuous, even if all of $\rho_{1}, \ldots, \rho_{n}$ are continuous; see Example 6. On the other hand, Example 15 below shows that monotonicity plus continuity from below is not sufficient for the inf-convolution of law-invariant risk functionals to be law-invariant.

Remark 3. Theorem 2 does not apply to atomic probability spaces such as a finite one. In Example 1, the law-invariant risk functionals $\rho_{1}$ and $\rho_{2}$ are actually monotone and uniformly continuous, but $\rho_{1} \square \rho_{2}$ is not law-invariant.

\section{Examples for law-invariance of inf-convolution}

Example 10 (Monetary risk measures). A monetary risk measure is a risk functional $\rho: \mathcal{X} \rightarrow \mathbb{R}$ satisfying monotonicity and cash-additivity. Special cases of monetary risk measures (up to sign change) include all commonly used risk measures, such as distortion risk measures (Yaari's dual utilities), convex risk measures, and coherent risk measures. For more on these risk measures, we refer to Föllmer and Schied (2016). Monotonicity and cash-invariance imply that $\rho$ is uniformly continuous. Hence, by Theorem 2 (i), as long as one of $\rho_{1}, \ldots, \rho_{n}$ is a law-invariant monetary risk measure, and the rest are law-invariant risk functionals, then $\square_{i=1}^{n} \rho_{i}$ is law-invariant and uniformly continuous. 
Remark 4. In the context of monetary risk measures, the law-invariance of $\rho$ (all risk measures here are assumed monetary) is equivalent to the acceptance set $\mathcal{A}_{\rho}=\{X \in \mathcal{X}: \rho(X) \leqslant 0\}$ being law-invariant. Since we can always write

$$
\rho_{1} \square \rho_{2}(X)=\inf \left\{m \in \mathbb{R}: X-m \in \mathcal{A}_{\rho_{1}}+\mathcal{A}_{\rho_{2}}\right\}
$$

for every $X \in \mathcal{X}$, the law-invariance of $\rho_{1} \square \rho_{2}$ is equivalent to the sup-norm closure of $\mathcal{A}_{\rho_{1}}+\mathcal{A}_{\rho_{2}}$ being law-invariant. Note that we have seen in Remark 1 that the sum of law-invariant sets is not necessarily law-invariant, whereas Example 10 implies that the sup-norm closure of the sum of law-invariant acceptance sets is law-invariant.

Example 11 (Mean-variance and mean-std functionals). A mean-variance functional, as originally used by Markowitz (1952) in portfolio theory, is defined as

$$
\rho(X)=\mathbb{E}[X]+\beta \operatorname{Var}(X), \quad X \in \mathcal{X}
$$

where $\beta>0$ is a constant. Similarly, one can define a mean-std functional, as

$$
\rho(X)=\mathbb{E}[X]+\beta \sqrt{\operatorname{Var}}(X), \quad X \in \mathcal{X} .
$$

A mean-variance or mean-std functional satisfies continuity and law-invariance (but not uniform continuity). Hence, it follows from (iii) of Theorem 2 that $\square_{i=1}^{n} \rho_{i}$ is law-invariant if one of $\rho_{1}, \ldots, \rho_{n}$ is a mean-variance (or mean-std) functional.

Example 12 (Expected utility maximization). A utility function is an increasing function $u: \mathbb{R} \rightarrow$ $[-\infty, \infty)$. For a utility function $u$, the expected utility functional is

$$
E_{u}(X)=\mathbb{E}[u(-X)], \quad X \in \mathcal{X}
$$

where $X$ is interpreted as a random loss. To be consistent with the choice of convention (minimization) in this paper, we can define the expected disutility functional by

$$
\rho(X)=-E_{u}(X), \quad X \in \mathcal{X}
$$

Clearly, if $u$ is continuous from below, then $\rho$ defined above is continuous from above and monotone. Therefore, by Theorem 2 (ii), if one of $\rho_{1}, \ldots, \rho_{n}$ is an expected disutility functional with $u$ continuous from below, and the others are law-invariant, then the inf-convolution $\square_{i=1}^{n} \rho_{i}$ is lawinvariant. 
Example 13 (Rank-dependent utilities). A rank-dependent utility (see, e.g., Quiggin (1982) and Quiggin (1993)) is defined as

$$
H_{u, h}(X)=\int_{0}^{\infty} h(\mathbb{P}(u(X)>x)) \mathrm{d} x+\int_{-\infty}^{0}(h(\mathbb{P}(u(X)>x))-1) \mathrm{d} x, \quad X \in \mathcal{X},
$$

where $u$ is a continuous utility function and $h$ is a distortion function (i.e. a continuous and increasing function on $[0,1]$ satisfying $h(0)=0$ and $h(1)=1)$. One can easily check that $H_{u, h}(X)$ is lawinvariant and continuous. In light of Theorem 2 (iii), the inf-convolution of law-invariant risk functionals is law-invariant if one of the risk functionals is a rank dependent utility. In particular, $\square_{i=1}^{n} H_{u_{i}, h_{i}}$ is law-invariant for all continuous utility functions $u_{1}, \ldots, u_{n}$ and distortion functions $h_{1}, \ldots, h_{n}$.

Example 14 (Cumulative prospect theory). A subjective utility in cumulative prospect theory (see, e.g., Tversky and Kahneman (1992)) is defined as the functional

$$
\rho(X)=\int_{-\infty}^{0} v(x) \mathrm{d} w_{1}\left(F_{X}(x)\right)-\int_{0}^{\infty} v(x) \mathrm{d} w_{2}\left(1-F_{X}(x)\right), \quad X \in \mathcal{X},
$$

where $v$ is a continuous utility function, and $w_{1}$ and $w_{2}$ are two distortion functions. One can easily check that $\rho$ is law-invariant and continuous. Hence, by (iii) of Theorem $2, \square_{i=1}^{n} \rho_{i}$ is lawinvariant as long as one of $\rho_{1}, \ldots, \rho_{n}$ is a subjective utility defined as in (18), and the others are law-invariant.

Example 15 (Monotonicity plus continuity from below is not sufficient). We revisit Example 2 in Section 3. Recall that in Example 2, we use two risk functionals

$$
\rho_{1}(X)=1-\mathbb{1}_{\left\{X \sim F_{B}\right\}} \text { and } \rho_{2}(X)=1-\mathbb{1}_{\left\{X \sim F_{U}\right\}}, \quad X \in \mathcal{X}
$$

where the distributions $F_{B}$ and $F_{U}$ are defined in Example 2. The above two risk functionals are not monotone or continuous. We can make the following modification

$$
\hat{\rho}_{1}(X)=1-\mathbb{1}_{\left\{X \preceq_{\mathrm{st}} F_{B}\right\}} \text { and } \hat{\rho}_{2}(X)=1-\mathbb{1}_{\left\{X \preceq_{\mathrm{st}} F_{U}\right\}}, \quad X \in \mathcal{X},
$$

where for a random variable $X$ and a distribution $G, X \preceq_{\text {st }} G$ stands for the usual stochastic order, that is, $\mathbb{P}(X \leqslant x) \geqslant G(x)$ for all $x \in \mathbb{R}$. With the above modification, we can check that both $\hat{\rho}_{1}$ and $\hat{\rho}_{2}$ are monotone and continuous from below.

Next, take the random variables $X$ and $Z$ as in Example 2. Obviously, $\hat{\rho}_{1} \square \hat{\rho}_{2}(X)=0$ since there exist $Y$ such that $Y \sim F_{B}$ and $X-Y \sim F_{U}$. Suppose for the purpose of contradiction that there exists $W \preceq_{\text {st }} F_{B}$ such that $Z-W \preceq_{\text {st }} F_{U}$. Note that $W \preceq_{\text {st }} F_{B}$ and $Z-W \preceq_{\text {st }} F_{U}$ imply 
$\mathbb{E}[W] \leqslant \frac{1}{2}$ and $\mathbb{E}[Z-W] \leqslant 0$. Since $\mathbb{E}[W]+\mathbb{E}[Z-W]=\mathbb{E}[Z]=\frac{1}{2}$, we know that $\mathbb{E}[W]=\frac{1}{2}$ and $\mathbb{E}[Z-W]=0$. Note that the partial order $\preceq_{\text {st }}$ together with equality in the mean implies equality in distribution. Therefore, $W \sim F_{B}$ and $Z-W \sim F_{U}$, which is not possible as verified in Example 2. Hence, $\hat{\rho}_{1} \square \hat{\rho}_{2}(Z) \geqslant 1>0=\hat{\rho}_{1} \square \hat{\rho}_{2}(X)$, and $\hat{\rho}_{1} \square \hat{\rho}_{2}$ is not law-invariant.

One interesting question about inf-convolution is exactness, that is, whether the infimum in an inf-convolution can be attained as a minimum. In the next example and the next remark, we discuss this issue, where we obtain a new observation on the sum of law-invariant acceptance sets.

Example 16 (Non-attainability of the inf-convolution). Following the same notation of Examples 2 and 15, we define two risk measures

$$
\rho_{1}(Y)=\inf \left\{m \in \mathbb{R}: Y-m \preceq_{\text {st }} F_{B}\right\} \text { and } \rho_{2}(Y)=\inf \left\{m \in \mathbb{R}: Y-m \preceq_{\text {st }} F_{U}\right\}, \quad Y \in \mathcal{X} .
$$

In other words, the acceptance set of $\rho_{1}$ is $\mathcal{A}_{1}=\left\{Y \in \mathcal{X}: Y \preceq_{\text {st }} F_{B}\right\}$ and that of $\rho_{2}$ is $\mathcal{A}_{2}=$ $\left\{Y \in \mathcal{X}: Y \preceq_{\text {st }} F_{U}\right\}$. Such $\rho_{1}$ and $\rho_{2}$ belong to the class of risk measures based on benchmark loss distributions studied by Bignozzi et al. (2019). One can easily check that $\rho_{1}$ and $\rho_{2}$ are both law-invariant monetary risk measures. Take the random variables $X$ and $Z$ as in Example 2. As we have seen in Example 2, there exists $Y \sim F_{B}$ such that $X-Y \sim F_{U}$, and hence $\rho_{1} \square \rho_{2}(X) \leqslant 0$ (in fact, one can check that $\rho_{1} \square \rho_{2}(X)=0$ ). By (i) of Theorem 2, $\rho_{1} \square \rho_{2}$ is law-invariant. Noting that $X$ and $Z$ have the same distribution function, we have $\rho_{1} \square \rho_{2}(Z)=\rho_{1} \square \rho_{2}(X) \leqslant 0$. If $\rho_{1} \square \rho_{2}(Z)$ is attained, then there exist $W \in \mathcal{X}$ and $x \in \mathbb{R}$ such that $\rho_{1}(W) \leqslant x$ and $\rho_{2}(Z-W) \leqslant-x$, which implies that $W-x \preceq_{\text {st }} F_{B}$ and $Z-W+x \preceq_{\text {st }} F_{U}$. As we have seen in Example 15, this leads to a contradiction. Therefore, the inf-convolution $\rho_{1} \square \rho_{2}(Z)$ cannot be attained.

Note that both $\rho_{1}$ and $\rho_{2}$ in Example 16 are monetary, which means that they are uniformly continuous; thus uniform continuity and law-invariance in Theorem 2 together do not guarantee exactness.

Remark 5. There are some results in the literature on the exactness of the inf-convolution of monetary risk measures, and Example 16 complements these results. We summarize these findings below. Let $\rho_{1}$ and $\rho_{2}$ be two monetary risk measures and $X \in \mathcal{X}$.

(i) If $\rho_{1} \square \rho_{2}(X)=-\infty$ (this actually implies that $\rho_{1} \square \rho_{2}=-\infty$ on $\mathcal{X}$ because of uniform continuity), then the inf-convolution cannot be exact at $X$. This is the case of VaR studied by Embrechts et al. (2018); see Example 4. 
(ii) If $\rho_{1} \square \rho_{2}(X)>-\infty$, and $\rho_{1}$ and $\rho_{2}$ are both convex risk measures, the inf-convolution may not be attained; see Exercise 21 in Delbaen (2012).

(iii) If $\rho_{1} \square \rho_{2}(X)>-\infty$, and $\rho_{1}$ and $\rho_{2}$ are both law-invariant convex risk measures, then the inf-convolution is exact at $X$. This is guaranteed by Theorem 10.52 of Rüschendorf (2013); see Example 3.

(iv) If $\rho_{1} \square \rho_{2}(X)>-\infty$, and $\rho_{1}$ and $\rho_{2}$ are both law-invariant and monetary risk measures, then inf-convolution may not be attained as shown in Example 16.

It is well known that the attainability of inf-convolution is equivalent to the $\|\cdot\|$-closedness of the sum of the corresponding acceptance sets. Example 16 implies that the sum of law-invariant acceptance sets is not necessarily closed; however, it is closed if one further assumes convexity of the acceptance sets, as suggested by (iii) of Remark 5 .

\section{Generalization beyond bounded random variables}

So far in this paper, we have considered the space $\mathcal{X}$ of bounded random variables as the common domain of risk functionals. In this section, we discuss the extension of our results to spaces beyond the space of bounded random variables.

For $p \in[0, \infty)$, let $L^{p}$ be the collection of all random variables having finite $p$-th moment defined on $(\Omega, \mathcal{F}, \mathbb{P})$. We treat the cases $L^{p}, p \in[0, \infty)$ and $L^{\infty}$ (the set of bounded random variables) separately because, generally, conclusions on inf-convolution for $L^{p}, p \in[0, \infty)$ and those for $L^{\infty}$ do not imply each other. This is due to the fact that the set $\mathcal{X}$ plays an important role in the definition of inf-convolution in (2)-(3), and a different choice of $\mathcal{X}$ for even the same risk functionals (restricted to smaller domains) may lead to a different value of the inf-convolution. We shall see this in the following example.

Example 17. Let $\rho_{1}(X)=\rho_{2}(X)=-\left(\sup \left\{p \geqslant 0: X \in L^{p}\right\}\right)^{-1}, X \in L^{0}$. One can easily check that $\rho_{1}(X)=\rho_{2}(X)=0$ for all $X \in L^{\infty}$. Therefore, if we set $\mathcal{X}=L^{\infty}$ in the definition of inf-convolution, then $\rho_{1} \square \rho_{2}(0)=0$. However, if we set $\mathcal{X}=L^{p}$, for some $0<p<\infty$, then $\rho_{1} \square \rho_{2}(0)=-2 / p$. Therefore, even for the same risk functionals, an analysis of its inf-convolution in the case $\mathcal{X}=L^{p}$ does not imply the case $\mathcal{X}=L^{\infty}$.

In the sequel, we fix $p \in[0, \infty)$ and set $\mathcal{X}=L^{p}$. A risk functional is a mapping from $\mathcal{X}$ to 
$[-\infty, \infty) .{ }^{5}$ We naturally define continuity and uniform continuity for risk functionals with respect to sup-norm $\|\cdot\|$, although $\|\cdot\|$ is no longer a norm on $L^{p}$. More precisely, we say that $\rho$ is continuous if $\rho\left(X_{n}\right) \rightarrow \rho(X)$ for any sequences of $\left\{X_{n}\right\}_{n \geqslant 1} \subset L^{p}$ and $X \in L^{p}$ satisfying $\left\|X_{n}-X\right\| \rightarrow 0$, and $\rho$ is uniformly continuous if for all $\varepsilon>0$ there exists $\delta>0$ such that for all $X, Y \in L^{p},\|X-Y\| \leqslant \delta$ implies $|\rho(X)-\rho(Y)| \leqslant \varepsilon$ with the convention $(-\infty)-(-\infty)=0$. We inherit all the other concepts and notation from the previous sections. Note that, although $\|\cdot\|$ is no longer a norm on $L^{p}$, all monetary risk measures on $L^{p}$ satisfy uniform continuity as we define above.

We first see that in the proof of Theorem 1, the boundedness of random variables is not relevant. Hence, the statement of Theorem 1 is valid for $\mathcal{X}=L^{p}$. Again, $\mathcal{X}^{\perp}$ is defined in (11).

Theorem 3. Let $\mathcal{X}=L^{p}, p \in[0, \infty)$. If $\rho_{1}, \ldots, \rho_{n}$ are law-invariant risk functionals, then $\square_{i=1}^{n} \rho_{i}$ is law-invariant on $\mathcal{X}^{\perp}$. In particular, if $\mathcal{X}=\mathcal{X}^{\perp}$, then $\square_{i=1}^{n} \rho_{i}$ is law-invariant.

Next, we generalize the results in Theorem 2 for $\mathcal{X}=L^{p}$ which requires a small modification to the proof of Theorem 2. Using Theorem 4, the examples in Section 6 are all valid on $L^{p}$, as long as the concerned risk functionals take finite values on $L^{p}$.

Theorem 4. Suppose that $\rho_{1}, \ldots, \rho_{n}$ are law-invariant risk functionals and $\mathcal{X}=L^{p}, p \in[0, \infty)$.

(i) If one of $\rho_{1}, \ldots, \rho_{n}$ is uniformly continuous, then $\square_{i=1}^{n} \rho_{i}$ is law-invariant and uniformly continuous.

(ii) If one of $\rho_{1}, \ldots, \rho_{n}$ is monotone and continuous from above, then $\square_{i=1}^{n} \rho_{i}$ is law-invariant, monotone and continuous from above.

(iii) If one of $\rho_{1}, \ldots, \rho_{n}$ is continuous, then $\square_{i=1}^{n} \rho_{i}$ is law-invariant.

Proof. Note that the statements in Lemmas 1-2 and Proposition 1 are still valid for $\mathcal{X}=L^{p}$, $p \in[0, \infty)$. Hence, cases (i) and (ii) follow from the same arguments as in the proof of Theorem 2. We next focus on case (iii). Without loss of generality, we assume that $\rho_{n}$ is continuous. Take $X, Y \in \mathcal{X}$ with $X \stackrel{\mathrm{d}}{=} Y$. Similarly to Theorem 2 , it suffices to show that for any $Z_{1}, \ldots, Z_{n-1} \in \mathcal{X}$, there exist sequences $\left\{W_{i, k}\right\}_{k \in \mathbb{N}} \subset \mathcal{X}, i=1, \ldots, n-1$ such that

$$
W_{i, k} \stackrel{\mathrm{d}}{=} Z_{i} \text { for all } i, k \text { and } \lim _{k \rightarrow \infty} \rho_{n}\left(Y-\sum_{i=1}^{n-1} W_{i, k}\right)=\rho_{n}\left(X-\sum_{i=1}^{n-1} Z_{i}\right)
$$

\footnotetext{
${ }^{5}$ Traditionally, a risk functional may take the value $\infty$ on $L^{p}$. However, for the problem inf-convolution this is irrelevant, as the possible allocations which lead to the value $\infty$ do not affect the minimization. Therefore, we exclude $\infty$ from the range of risk functionals.
} 
Write $Z=\sum_{i=1}^{n-1} Z_{i}$ and fix $k \in \mathbb{N}$. Again, using the same argument in the proof of Theorem 2, we know that there exist disjoint sets $A_{\ell}, \ell \in \mathbb{Z}$ such that for all integers $j \in \mathbb{Z}$,

$$
\mathbb{P}\left(\left\{Y \leqslant \frac{j}{k}\right\} \cap A_{\ell}\right)=\mathbb{P}\left(\left\{X \leqslant \frac{j}{k}\right\} \cap\left\{\frac{\ell}{k}<Z \leqslant \frac{\ell+1}{k}\right\}\right)
$$

$\mathbb{P}\left(A_{\ell}\right)=\mathbb{P}\left(\frac{\ell}{k}<Z \leqslant \frac{\ell+1}{k}\right)$, and $\mathbb{1}_{A_{\ell}} \in \mathcal{X}^{\perp}$. Hence, for $\ell \in \mathbb{Z}$, there exists a random vector $\left(W_{1, k}^{(\ell)}, \ldots, W_{n-1, k}^{(\ell)}\right)$ independent of $A_{\ell}$ such that

$$
\mathbb{P}\left(\left(W_{1, k}^{(\ell)}, \ldots, W_{n-1, k}^{(\ell)}\right) \leqslant \mathbf{w}\right)=\mathbb{P}\left(\left(Z_{1}, \ldots, Z_{n-1}\right) \leqslant \mathbf{w} \mid \frac{\ell}{k}<Z \leqslant \frac{\ell+1}{k}\right) \text { for all } \mathbf{w} \in \mathbb{R}^{n-1} .
$$

Define random variables $W_{i, k}, i=1, \ldots, n-1$, and $W_{k}$ by

$$
W_{i, k}=\sum_{\ell \in \mathbb{Z}} \mathbb{1}_{A_{\ell}} W_{i, k}^{(\ell)} \quad \text { and } \quad W_{k}=\sum_{i=1}^{n-1} W_{i, k}
$$

which satisfy $W_{i, k} \stackrel{\mathrm{d}}{=} Z_{i}, i=1, \ldots, n-1$. With $W_{i, k}$ defined above, we can follow the same argument as in the proof of Theorem 2, and obtain that (15) and (16) hold. This further implies (17), which now reads as $\left|F_{Y-W_{k}}^{-1}(U)-F_{X-Z}^{-1}(U)\right| \leqslant 4 / k$, where $U$ is a uniform random variable taking values on $[0,1]$. Hence, by the assumed continuity of $\rho_{n}$,

$$
\lim _{k \rightarrow \infty} \rho_{n}\left(Y-W_{k}\right)=\lim _{k \rightarrow \infty} \rho_{n}\left(F_{Y-W_{k}}^{-1}(U)\right)=\rho_{n}\left(F_{X-Z}^{-1}(U)\right)=\rho_{n}(X-Z) .
$$

This completes the proof.

Remark 6. We finally discuss the possibility of generalizing our main results to spaces beyond $L^{p}$. Assume that $\mathcal{X}$ is a generic convex cone containing $L^{\infty}$. The results in Lemma 1, Lemma 3, Proposition 1 and Theorem 4 (i)-(ii) do not require any specific structure of $\mathcal{X}$, and hence they are still valid. For Theorem 3 and the arguments in the proof of Theorem 4 (iii) to work, it suffices to assume that $\mathcal{X}$ is law-invariant (meaning that $X \in \mathcal{X}$ and $X \stackrel{\mathrm{d}}{=} Y$ implies $Y \in \mathcal{X}$ ).

\section{Acknowledgements}

The authors thank the Editor, three anonymous referees, Tim Boonen, and Alexander Schied for their very helpful comments. R. Wang acknowledges financial support from the Natural Sciences and Engineering Research Council of Canada (NSERC, RGPIN-2018-03823, RGPAS-2018-522590) and from the Center of Actuarial Excellence Research Grant from the Society of Actuaries. L. Wei acknowledges financial support from the China Scholarship Council. 


\section{References}

Arrow, K.J. (1974). Essays in the Theory of Risk-bearing. North-Holland, New York.

Artzner, P., Delbaen, F., Eber, J.-M. and Heath, D. (1999). Coherent measures of risk. Mathematical Finance, 9(3), 203-228.

Barrieu, P. and El Karoui, N. (2005). Inf-convolution of risk measures and optimal risk transfer. Finance and Stochastics, 9, 269-298.

Bellini, F. and Bignozzi, V. (2015). On elicitable risk measures. Quantitative Finance, 15(5), 725-733.

Bernard, C., Jiang, X. and Wang, R. (2014). Risk aggregation with dependence uncertainty. Insurance: Mathematics and Economics, 54, 93-108.

Bignozzi, V., Burzoni, M. and Munari, C. (2019). Risk measures based on benchmark loss distributions. Journal of Risk and Insurance, published online.

Billingsley, P. (1995). Probability and Measure. John Wiley \& Sons, Inc., New York, Third Edition.

Chen, S., Gao, N. and Xanthos, F. (2018). The strong Fatou property of risk measures. Dependence Modeling, 6(1), 183-196.

Cont, R., Deguest, R. and Scandolo, G. (2010). Robustness and sensitivity analysis of risk measurement procedures. Quantitative Finance, 10(6), 593-606.

Delbaen, F. (2002). Coherent risk measures on general probability spaces. In Advances in Finance and Stochastics (pp. 1-37). Springer, Berlin, Heidelberg.

Delbaen, F. (2012). Monetary Utility Functions. Osaka University Press, Osaka.

Denneberg, D. (1994). Non-additive Measure and Integral. Kluwer, Dordrecht.

Embrechts, P., Liu, H. and Wang, R. (2018). Quantile-based risk sharing. Operations Research, 66(4), 936949.

Embrechts, P., Liu, H., Mao, T. and Wang, R. (2019). Quantile-based risk sharing with heterogeneous beliefs. Mathematical Programming Series B, published online.

Embrechts, P., Puccetti, G. and Rüschendorf, L. (2013). Model uncertainty and VaR aggregation. Journal of Banking and Finance, 37(8), 2750-2764.

Embrechts, P., Puccetti, G., Rüschendorf, L., Wang, R. and Beleraj, A. (2014). An academic response to Basel 3.5. Risks, 2(1), 25-48.

Embrechts, P., Wang, B. and Wang, R. (2015). Aggregation-robustness and model uncertainty of regulatory risk measures. Finance and Stochastics, 19(4), 763-790.

Filipović, D. and Svindland, G. (2008). Optimal capital and risk allocations for law- and cash-invariant convex functions. Finance and Stochastics, 12, 423-439.

Föllmer, H. and Schied, A. (2016). Stochastic Finance. An Introduction in Discrete Time. Walter de Gruyter, Berlin, Fourth Edition.

Gao, N. Leung, D., Munari, C. and Xanthos, F. (2018). Fatou property, representations, and extensions of law-invariant risk measures on general Orlicz spaces. Finance and Stochastics, 22, 395-415. 
Jouini, E., Schachermayer, W. and Touzi, N. (2006). Law invariant risk measures have the Fatou property. Advances in Mathematical Economics, 9, 49-71.

Jouini, E., Schachermayer, W. and Touzi, N. (2008). Optimal risk sharing for law invariant monetary utility functions. Mathematical Finance, 18(2), 269-292.

Karatzas, I. and Shreve, E. (1991). Brownian Motion and Stochastic Calculus. Springer-Verlag, New York, Second Edition.

Kusuoka, S. (2001). On law invariant coherent risk measures. Advances in Mathematical Economics, 3, 83-95.

Mao, T. and Wang, R. (2016). Risk aversion in regulatory capital calculation. SSRN: 2658669.

Markowitz, H. (1951). Portfolio selection, Journal of Finance, 7(1), 77-91.

Quiggin, J. (1982). A theory of anticipated utility. Journal of Economic Behavior E Organization , 3(4), $323-343$.

Quiggin, J. (1993). Generalized Expected Utility Theory: The Rank-dependent Model. Kluwer, the Netherlands.

Rockafellar, R. T., Uryasev, S. and Zabarankin, M. (2006). Generalized deviation in risk analysis. Finance and Stochastics, 10, 51-74.

Rüschendorf, L. (2013). Mathematical Risk Analysis. Dependence, Risk Bounds, Optimal Allocations and Portfolios. Springer, Heidelberg.

Starr, R. M. (2011). General Equilibrium Theory: An Introduction. Second Edition, Cambridge University Press.

Tsanakas, A. (2009). To split or not to split: Capital allocation with convex risk measures. Insurance: Mathematics and Economics, 44(2), 268-277.

Tversky, A. and Kahneman, E. (1992). Advances in prospect theory: cumulative representation of uncertainty. Journal of Risk and Uncertainty, 5, 297-323.

Wang, R. and Wei, Y. (2018). Characterizing optimal allocations in quantile-based risk sharing. SSRN: 3173503.

Wang, S., Young, V. R. and Panjer, H. H. (1997). Axiomatic characterization of insurance prices. Insurance: Mathematics and Economics, 21(2), 173-183.

Weber, S. (2006). Distribution-invariant risk measures, information, and dynamic consistency. Mathematical Finance, 16, 419-441.

Weber, S. (2018). Solvency II, or how to sweep the downside risk under the carpet. Insurance: Mathematics and Economics, 82, 191-200.

Ziegel, J. (2016). Coherence and elicitability. Mathematical Finance, 26, 901-918. 